\title{
A Common Fixed Point Theorem in Menger Space using the Property CLR
}

\author{
Reema Singh ${ }^{1}$, Bharat Singh ${ }^{2}$, Amrish Singh ${ }^{3 .}$ \\ ${ }^{I}$ (Department of Mathematics and Statistics, Medicaps University, Indore, M.P., India) \\ ${ }^{2}$ (School of Computers \& Electronics, IPS Academy, Indore, M.P, India) \\ ${ }^{3}$ (Department of Mathematics, Saint Thomas School, Dewas, M.P, India)
}

\begin{abstract}
The purpose of this paper is to obtain a common fixed point theorem in a Menger space for weakly compatible self-map satisfying a contractive condition by using an implicit relation and the property CLR which will also generalize and improve several results on fixed point in probabilistic metric spaces and menger spaces.
\end{abstract}

Keywords: Common fixed point, distribution function, Menger space, $t$-norm, weakly compatible mapping.

\section{Introduction}

Fixed point theory in Menger spaces can be considered as a part of Probabilistic Analysis, which is a very dynamic area of mathematical research. The notion of probabilistic metric space is introduced by Menger in 1942 [8] and the first result about the existence of a fixed point of a mapping which is defined on a Menger space is obtained by Sehgel and Barucha-Reid. Recently, a number of fixed point theorems for single valued and multivalued mappings in Menger probabilistic metric space have been considered by many authors $[1],[2],[3],[4],[5],[6]$.

In 1998, Jungck [7] introduced the concept weakly compatible maps and proved many theorems in metric space.

Further, the study of common fixed point of mapping satisfying contractive type conditions has been a very active field of research activity during the last three decades. Many authors have proved a number of fixed point theorems for different contractions in Menger Spaces.

And, most recently, Sintunavarat and Kumam [9] defined the notion of "Common limit in the range" property or CLR property in fuzzy metric spaces

In this note, we prove a common fixed point theorem for four mappings with weak compatibility satisfying a new contractive condition without appeal to continuity in Menger space using contractive condition with the notion of CLR property.

\section{Preliminaries}

Definition 1[10] Suppose that $(X, d)$ is a metric space and $f, g: X \rightarrow X$. The pair of mappings $(f, g)$ is said to satisfy the common limit in the range of $g$ property if there exists a sequence $\left\{x_{n}\right\}$ in $X$ such that $\lim _{n \rightarrow \infty} f\left(x_{n}\right)=\lim _{n \rightarrow \infty} g\left(x_{n}\right)=g(x)$ for some $x \in X$.

Similarly we can have the property $\left(\mathrm{CLR}_{\mathrm{T}}\right)$ and the property $\left(\mathrm{CLR}_{\mathrm{S}}\right)$ if mapping $\mathrm{f}$ and $\mathrm{g}$ is replaced by $\mathrm{T}$ and $\mathrm{S}$ respectively.

Definition 2 [11] A mapping $F: \mathbb{R} \rightarrow \mathbb{R}^{+}$is called a distribution function if it is non-decreasing and left continuous with $\inf _{t \in \mathbb{R}} F(t)=0$ and $\sup _{t \in \mathbb{R}} F(t)=1$ where $\mathbb{R}$ is the set of real numbers and $\mathbb{R}^{+}$denotes the set of non-negative real numbers.

Definition 3 [11] A Binary operation $\Delta:[0,1]^{2} \rightarrow[0,1]$ is called a $t-$ norm if it satisfies the following properties:

(i) $\Delta$ is associative and commutative

(ii) $\Delta(a, 1)=a \forall a \in[0,1]$,

(iii) $\Delta(a, b) \leq \Delta(c, d)$ whenever $a \leq c$ and $b \leq d \forall a, b, c, d \in[0,1]$.

Definition 4 [11] A Menger Space is a triplet $(X, F, \Delta)$, where $X$ is a non-empty space, $\mathrm{F}$ is a function defined on $X \times X$ to the set of distribution functions and $\Delta$ is a $t-n o r m$ such that the following properties are satisfied:

(i) $F_{x, y}(0)=0 \forall x, y \in X$,

(ii) $F_{x, y}(s)=1 \forall s>0$ if and only if $x=y$,

(iii) $F_{x, y}(s)=F_{y, x}(s) \forall s>0, x, y \in X$ 
(iv) $F_{x, y}(u+v) \geq \Delta\left(F_{x, z}(u), F_{z, y}(v)\right) \forall u, v \geq 0, x, y, z \in X$.

Definition 5 [12] Let $(X, F, \Delta)$ be a Menger Space.

(i) A sequence $\left\{x_{n}\right\}$ in $X$ is said to be convergent to a point $x \in X$ if $\lim _{n \rightarrow \infty} F_{x_{n}, x}(t)=1 \forall t>0$.

(ii) A sequence $\left\{x_{n}\right\}$ in $X$ is said to be a Cauchy sequence if for each $0<\varepsilon<1$ and $t>0$, there cexist a positive integer $n_{0}$ such that $F_{x_{n}, x_{m}}(t)>1-\varepsilon$ for each $n, m \geq n_{0}$

(iii) A Menger Space in which every Cauchy sequence is convergent is said to be complete.

Definition $6[13]$ The 3-tuple $(X, M, \Delta)$ is called a fuzzy metric space if $X$ is an arbitrary set, $\Delta$ is a continuous $\mathrm{t}$-norm and $\mathrm{M}$ is a fuzzy set in $\mathrm{X}^{2} \times(0, \infty)$ satisfying the following conditions for all $\mathrm{x}, \mathrm{y}, \mathrm{z} \in \mathrm{X}$ and $\mathrm{t}, \mathrm{s}>0$.

(i) $M(x, y, 0)>0$

(ii) $M(x, y, t)=1, \forall t>0$ iff $x=y$

(iii) $M(x, y, t)=M(y, x, t)$,

(iv) $\Delta(M(x, y, t), M(y, z, s)) \leq M(x, z, t+s)$

(v) $\mathrm{M}(\mathrm{x}, \mathrm{y},):.[0, \infty) \rightarrow[0,1]$ is left continuous

(vi) $\lim _{t \rightarrow \infty} M(x, y, t)=1$.

Definition 7 [7] The mappings $f, g: X \rightarrow X$ are said to be weakly compatible if $f(g(x))=g(f(x)) \forall x \in X$ such that $f(x)=g(x)$.

Definition 8[15] Let $(X, F, \Delta)$ be a Menger Space with a continuous $t-$ norm $\Delta$. The two mappings $f, g: X \rightarrow$ $X$ are said to have the CLRg property if there exists a sequence $\left\{x_{n}\right\}$ in $X$ and a point $z$ in $X$ such that $\lim _{n \rightarrow \infty} f\left(x_{n}\right)=\lim _{n \rightarrow \infty} g\left(x_{n}\right)=g(z)$.

Definition $9 \mathrm{We}$ will apply an implicit relation as, let $\Phi_{5}$ denote the set of all continuous functions from $[0,1]^{5} \rightarrow \mathrm{R}$ satisfying the conditions:

$\Phi_{1}$ : $\Phi$ is non-increasing in $t_{2}, t_{3}, t_{4}$ and $t_{5}$

$\Phi_{2}: \Phi(\mathrm{u}, \mathrm{v}, \mathrm{v}, \mathrm{v}, \mathrm{v}) \geq 0 \Longrightarrow \mathrm{u} \geq \mathrm{v}$ for $\mathrm{u}, \mathrm{v} \in[0,1]$

Example: $\Phi\left(\mathrm{t}_{1}, \mathrm{t}_{2}, \mathrm{t}_{3}, \mathrm{t}_{4}, \mathrm{t}_{5}\right)=\mathrm{t}_{1}-\max \left\{\mathrm{t}_{2}, \mathrm{t}_{3}, \mathrm{t}_{4}, \mathrm{t}_{5}\right\}$

Lemma1 [14] Let $(X, F, \Delta)$ be a Menger Space and $x, y \in X$. If there exists a constant $k \in(0,1)$ such that $F_{x, y}(k t) \geq F_{x, y}(t)$ for all $t>0$, then $x=y$.

\section{Main Result}

Theorem: Let A, B, S and T be self- maps of a Menger space $(X, F, \Delta)$ satisfying the following conditions:

(i) $\mathrm{A}(\mathrm{X}) \subseteq \mathrm{T}(\mathrm{X}), \mathrm{B}(\mathrm{X}) \subseteq \mathrm{S}(\mathrm{X})$

(ii) $(\mathrm{A}, \mathrm{S})$ and $(\mathrm{B}, \mathrm{T})$ are weakly compatible pairs

(iii) $(A, S)$ or $(B, T)$ satisfy the property $\left(C L R_{S}\right)$ and $\left(C_{L} R_{T}\right)$ respectively.

(iv) For some $\Phi \in \Phi_{5}$, and for all $\mathrm{x}, \mathrm{y} \in \mathrm{X}, \mathrm{t}>0 \& \mathrm{k} \in(0,1)$,

$$
\varphi\left[F_{A x, B y}(k t), F_{S x, T y}(t), \frac{F_{B y, S x}(t)+F_{B y, T y}(t)}{2}, \frac{F_{A x, T y}(t)+F_{S x, A x}(t)}{2}, F_{S x, A x}(t)\right] \geq 0
$$

If the range of one of the maps $\mathrm{A}, \mathrm{B}, \mathrm{S}$ or $\mathrm{T}$ is a complete subspace of $\mathrm{X}$. then $\mathrm{A}, \mathrm{B}, \mathrm{S}$ and $\mathrm{T}$ have a unique common fixed point in $\mathrm{X}$.

Proof: Without loss of generality, assume that $B(X) \subseteq S(X)$ and the pair $(\mathrm{B}, \mathrm{T})$ satisfies property $\left(\mathrm{CLR}_{\mathrm{T}}\right)$, then there exist a sequence $\left\{x_{n}\right\}$ in $X$ such that $B x_{n}$ and $\mathrm{Tx}_{\mathrm{n}}$ converges to $\mathrm{Tx}$, for some $\mathrm{x}$ in $\mathrm{X}$ as $\mathrm{n} \rightarrow \infty$. Since $B(X) \subseteq S(X)$, so there exist a sequence $\left\{\mathrm{y}_{\mathrm{n}}\right\}$ in $\mathrm{X}$ such that $\mathrm{Bx}_{\mathrm{n}}=S \mathrm{Sy}_{\mathrm{n}}$, hence $\mathrm{Sy}_{\mathrm{n}} \rightarrow \mathrm{Tx}$ as $\mathrm{n} \rightarrow \infty$. We shall show that $\lim _{\mathrm{n} \rightarrow \infty} A y_{n}=\mathrm{Tx}$.

Suppose $\mathrm{Ay}_{\mathrm{n}} \rightarrow \mathrm{w}(\neq \mathrm{Tx})$ then by taking $\mathrm{x}=\mathrm{y}_{\mathrm{n}}, \mathrm{y}=\mathrm{x}_{\mathrm{n}}$ in (iv)

$$
\varphi\left[F_{A y_{\mathrm{n}}, B \mathrm{x}_{\mathrm{n}}}(k t), F_{S \mathrm{yn}_{\mathrm{n}}, T \mathrm{x}_{\mathrm{n}}}(t), \frac{F_{B \mathrm{x}_{\mathrm{n}}, S \mathrm{yn}_{\mathrm{n}}}(t)+F_{B \mathrm{x}_{\mathrm{n}}, T \mathrm{x}_{\mathrm{n}}}(t)}{2}, \frac{F_{A \mathrm{yn}_{\mathrm{n}}, T \mathrm{x}_{\mathrm{n}}}(t)+F_{S \mathrm{yn}_{\mathrm{n}}, A \mathrm{y}_{\mathrm{n}}}(t)}{2}, F_{S \mathrm{y}_{\mathrm{n}}, A \mathrm{y}_{\mathrm{n}}}(t)\right] \geq 0
$$

Letting $\mathrm{n} \rightarrow \infty$ we get

$\varphi\left[F_{w, T x}(k t), F_{T x, T x}(t), \frac{F_{T x, T x}(t)+F_{T x, T x}(t)}{2}, \frac{F_{w, T x}(t)+F_{T x, w}(t)}{2}, F_{T x, w}(t)\right] \geq 0$ 
$\Rightarrow \varphi\left[F_{w, T x}(k t), 1,1, F_{w, T x}(t), F_{T x, w}(t)\right] \geq 0$

$\Rightarrow \varphi\left[F_{w, T x}(k t), F_{w, T x}(t), F_{w, T x}(t), F_{w, T x}(t), F_{w, T x}(t)\right] \geq 0$ since $\Phi$ is non-increasing in $t_{2}$ and $t_{3}$

Therefore using the property $\varphi_{2}$ (in definition 1.9), we have $F_{w, T x}(k t) \geq F_{w, T x}(t)$.

Thus by using lemma 1 we have $T x=w$

This shows that $A y_{n} \rightarrow T x$ as $n \rightarrow \infty$.

Now suppose that $S(X)$ is a complete subspace of $X$ then $T x=S u$ for some $u \in X$. Subsequently we have, $\mathrm{A}_{\mathrm{n}} \rightarrow \mathrm{Su}, \mathrm{Bx}_{\mathrm{n}} \rightarrow \mathrm{Su}, \mathrm{Tx}_{\mathrm{n}} \rightarrow \mathrm{Su}$ and $\mathrm{Sy}_{\mathrm{n}} \rightarrow \mathrm{Su}$ as $\mathrm{n} \rightarrow \infty$

Taking $x=u, y=x_{n}$ in (iv), we have,

$\varphi\left[F_{A \mathrm{u}, B \mathrm{x}_{\mathrm{n}}}(k t), F_{S \mathrm{u}, T \mathrm{x}_{\mathrm{n}}}(t), \frac{F_{B \mathrm{x}_{\mathrm{n}}, S \mathrm{u}}(t)+F_{B \mathrm{x}_{\mathrm{n}}, T \mathrm{x}_{\mathrm{n}}}(t)}{2}, \frac{F_{A \mathrm{u}, T \mathrm{x}_{\mathrm{n}}}(t)+F_{S \mathrm{u}, A \mathrm{u}}(t)}{2}, F_{S \mathrm{u}, A \mathrm{u}}(t)\right] \geq 0$

Letting $\mathrm{n} \rightarrow \infty$ we get

$\varphi\left[F_{A u, S u}(k t), F_{S u, S u}(t), \frac{F_{S u, S u}(t)+F_{S u, S u}(t)}{2}, \frac{F_{A u, S u}(t)+F_{S u, A u}(t)}{2}, F_{S u, A u}(t)\right] \geq 0$

$\Rightarrow \varphi\left[F_{A \mathrm{u}, S \mathrm{u}}(k t), 1,1, F_{A \mathrm{u}, S \mathrm{u}}(t), F_{S \mathrm{u}, A \mathrm{u}}(t)\right] \geq 0$

$\Rightarrow \varphi\left[F_{A u, S u}(k t), F_{A u, S u}(t), F_{A u, S u}(t), F_{A u, S u}(t), F_{S u, A u}(t)\right] \geq 0$ since $\Phi$ is non-increasing in $\mathrm{t}_{2}$ and $\mathrm{t}_{3}$.

Therefore using the property $\varphi_{2}$ (in definition 1.9), we have $F_{A \mathrm{u}, S \mathrm{u}}(k t) \geq F_{A \mathrm{u}, S \mathrm{u}}(t)$.

Thus by using lemma 1 we have $\mathrm{Au}=\mathrm{Su}$

But weak compatibility of A and S implies ASu $=\mathrm{SAu}$

Hence $\mathrm{AAu}=\mathrm{ASu}=\mathrm{SSu}$.

On the other hand since $A(\mathrm{X}) \subseteq \mathrm{T}(\mathrm{X})$, therefore there exist $\mathrm{u}, \mathrm{v} \in \mathrm{X}$. such that $\mathrm{Au}=\mathrm{Tv}$.

Now we show that $\mathrm{Tv}=\mathrm{Bv}$.

Taking $\mathrm{x}=\mathrm{u}, \mathrm{y}=\mathrm{v}$ in (iv), we have $\varphi\left[F_{A \mathrm{u}, B v}(k t), F_{S \mathrm{u}, T v}(t), \frac{F_{B v, S u}(t)+F_{B v}, T v}{2}(t), \frac{F_{A \mathrm{u}, T v}(t)+F_{S \mathrm{u}, A \mathrm{u}}(t)}{2}, F_{S \mathrm{u}, A \mathrm{u}}(t)\right] \geq$ 0

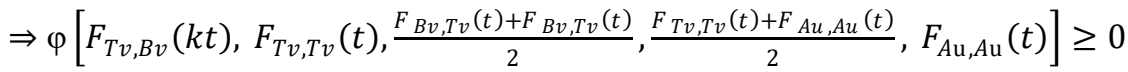

$\Rightarrow \varphi\left[F_{T v, B v}(k t), 1, F_{T v, B v}(t), 1,1\right] \geq 0$

$\Rightarrow \varphi\left[F_{T v, B v}(k t), F_{T v, B v}(t), F_{T v, B v}(t), F_{T v, B v}(t), F_{T v, B v}(t)\right] \geq 0$ since $\Phi$ is non-increasing in $\mathrm{t}_{2}, \mathrm{t}_{4}$ and $\mathrm{t}_{5}$.

Therefore using the property $\varphi_{2}$ (in definition 1.9), we have $F_{T v, B v}(k t) \geq F_{T v, B v}(t)$.

Thus by using lemma 1 we have $\mathrm{Tv}=\mathrm{Bv}$.

Hence $\mathrm{Au}=\mathrm{Su}=\mathrm{Tv}=\mathrm{Bv}$.

But the weak compatibility of B and T implies that $\mathrm{BTv}=\mathrm{TBv}$

Hence $\mathrm{TBv}=\mathrm{BTv}=\mathrm{BBv}$.

Now let us show that $\mathrm{Au}$ is a common fixed point of A, B, S and T.

Taking $\mathrm{x}=\mathrm{Au}, \mathrm{y}=\mathrm{u}$ in (iv), it follows

$\varphi\left[F_{A A \mathrm{u}, A u}(k t), F_{A A u, A u}(t), \frac{F_{A u, A A u}(t)+F_{A u, A u}(t)}{2}, \frac{F_{A A \mathrm{u}, A u}(t)+F_{A \mathrm{u}, A \mathrm{u}}(t)}{2}, F_{A A u, A A \mathrm{u}}(t)\right] \geq 0$

$\Rightarrow \varphi\left[F_{A A u, A u}(k t), F_{A A u, A u}(t), \frac{F_{A u, A A u}(t)+1}{2}, \frac{F_{A A u, A u}(t)+1}{2}, 1\right] \geq 0$

$\Rightarrow \varphi\left[F_{A A u, A u}(k t), F_{A A u, A u}(t), F_{A A u, A u}(t), F_{A A u, A u}(t), 1\right] \geq 0$

$\Rightarrow \varphi\left[F_{A A u, A u}(k t), F_{A A u, A u}(t), F_{A A u, A u}(t), F_{A A u, A u}(t), F_{A A u, A u}(t)\right] \geq 0$ since $\Phi$ is non-increasing in $\mathrm{t}_{5}$.

Therefore using the property $\varphi_{2}$ (in definition 1.9), we have $F_{A A u, A u}(k t) \geq F_{A A u, A u}(t)$.

Thus by using lemma 1 , we have $\mathrm{AAu}=\mathrm{Au}$.

Hence $A u=A A u=S A u$ and $A u$ is a common fixed point of $A$ and $S$.

Similarly we can prove that $\mathrm{Bv}$ is a common fixed point of $\mathrm{B}$ and $\mathrm{T}$.

Now since $\mathrm{Au}=\mathrm{Bv}$, we conclude that $\mathrm{Au}$ is a common fixed point of $\mathrm{A}, \mathrm{B}, \mathrm{S}$ and $\mathrm{T}$.

The proof is similar when $\mathrm{T}(\mathrm{X})$ is assumed to be complete subspace of $\mathrm{X}$. The cases in which $\mathrm{A}(\mathrm{X})$ or $\mathrm{B}(\mathrm{X})$ is complete subspace of $X$ are similar to the cases in which $\mathrm{T}(\mathrm{X})$ or $\mathrm{S}(\mathrm{X})$ respectively is complete since $\mathrm{A}(\mathrm{X}) \subseteq$ $\mathrm{T}(\mathrm{X})$ and $\mathrm{B}(\mathrm{X}) \subseteq \mathrm{S}(\mathrm{X})$.

For uniqueness of fixed point, let $\mathrm{Au}=\mathrm{Bu}=\mathrm{Tu}=\mathrm{Su}=\mathrm{w}_{1}$ and $\mathrm{Av}=\mathrm{Bv}=\mathrm{Tv}=\mathrm{Sv}=\mathrm{w}_{2}$ 
Then using (iv), we have

$$
\begin{aligned}
& \varphi\left[F_{A \mathrm{u}, B v}(k t), F_{S \mathrm{u}, T v}(t), \frac{F_{B v, S \mathrm{u}}(t)+F_{B v, T v}(t)}{2}, \frac{F_{A \mathrm{u}, T v}(t)+F_{S \mathrm{u}, A \mathrm{u}}(t)}{2}, F_{S \mathrm{u}, A \mathrm{u}}(t)\right] \geq 0 \\
& \Rightarrow \varphi\left[F_{\mathrm{w}_{1}, \mathrm{w}_{2}}(k t), F_{\mathrm{w}_{1}, \mathrm{w}_{2}}(t), \frac{F_{\mathrm{w}_{2}, \mathrm{w}_{1}}(t)+F_{\mathrm{w}_{2}, \mathrm{w}_{2}}(t)}{2}, \frac{F_{\mathrm{w}_{1}, \mathrm{w}_{2}}(t)+F_{\mathrm{w}_{1}, \mathrm{w}_{1}}(t)}{2}, F_{\mathrm{w}_{1}, \mathrm{w}_{1}}(t)\right] \geq 0 \\
& \Rightarrow \varphi\left[F_{\mathrm{w}_{1}, \mathrm{w}_{2}}(k t), F_{\mathrm{w}_{1}, \mathrm{w}_{2}}(t), \frac{F_{\mathrm{w}_{2}, \mathrm{w}_{1}}(t)+1}{2}, \frac{F_{\mathrm{w}_{1}, \mathrm{w}_{2}}(t)+1}{2}, 1\right] \geq 0 \\
& \Rightarrow \varphi\left[F_{\mathrm{w}_{1}, \mathrm{w}_{2}}(k t), F_{\mathrm{w}_{1}, \mathrm{w}_{2}}(t), F_{\mathrm{w}_{1}, \mathrm{w}_{2}}(t), F_{\mathrm{w}_{1}, \mathrm{w}_{2}}(t), 1\right] \geq 0 \\
& \Rightarrow \varphi\left[F_{\mathrm{w}_{1}, \mathrm{w}_{2}}(k t), F_{\mathrm{w}_{1}, \mathrm{w}_{2}}(t), F_{\mathrm{w}_{1}, \mathrm{w}_{2}}(t), F_{\mathrm{w}_{1}, \mathrm{w}_{2}}(t), F_{\mathrm{w}_{1}, \mathrm{w}_{2}}(t)\right] \geq 0 \text { since } \Phi \text { is non-increasing in } \mathrm{t}_{5} .
\end{aligned}
$$

Therefore using the property $\varphi_{2}$ (in definition 1.9), we have $F_{\mathrm{w}_{1}, \mathrm{w}_{2}}(k t) \geq F_{\mathrm{w}_{1}, \mathrm{w}_{2}}(t)$.

Thus by using lemma 1 we have $\mathrm{w}_{1}=\mathrm{w}_{2}$.

Hence the common fixed point is unique.

\section{Conclusion}

The result on a common fixed point is successfully obtained for weakly compatible maps in a Menger space using the common limit in the range property of mappings called (CLR) property. Moreover the result does not require the continuity of maps and improves several results on fixed point in probabilistic metric spaces and menger spaces.

\section{References}

[1]. A.T.Bharucha Ried, Fixed point theorems in Probabilistic analysis, Bull. Amer.Math. Soc, 82 (1976), $611-617$.

[2]. Gh.Boscan, On some fixed point theorems in Probabilistic metric spaces, Math.balkanica, 4 (1974), 67-70.

[3]. S. Chang, Fixed points theorems of mappings on Probabilistic metric spaces with applications, Scientia Sinica SeriesA, 25 (1983), 114-115.

[4]. R. Dedeic and N. Sarapa, Fixed point theorems for sequence of mappings on Menger spaces, Math. Japonica, 34 (4) (1988), 535539.

[5]. O.Hadzic, On the ( $\varepsilon, \lambda)$-topology of LPC-Spaces, Glasnik Mat; 13(33) (1978), 293-297.

[6]. O.Hadzic, Some theorems on the fixed points in probabilistic metric and random normed spaces, Boll. Un. Mat. Ital; 13(5) 18 (1981), 1-11

[7]. G.Jungck and B.E. Rhodes, Fixed point for set valued functions without continuity, Indian J. Pure.Appl. Math., 29(3) (1998), 977983.

[8]. K. Menger, Statistical Matrices, Procedings of the National academy of sciences of the United States of America 28 (1942), 535537.

[9]. Sintunavarat, W. and Kumam, P.: Common fixed point theorems for a pair of weakly compatible mappings in fuzzy metric spaces, J. Appl. Math, vol-2011, Article ID 637958, 14 pages (2011)

[10]. W. Sintunavarat and P. Kumam, Common fixed point theorems for a pair of weakly compatible mappings in fuzzy metric spaces, J. Appl. Math. 2011, Art. ID 637958.

[11]. B. Schweizer and A. Sklar, Probabilistic Metric Spaces, Elsevier, North-Holland 1983.

[12]. B. Schweizer, A. Sklar and E. Thorp, The metrization of statistical metric spaces, Pac. J.Math. 10 (1960) 673-675.

[13]. I. Kramosil and J. Michalek, Fuzzy metric and statistical metric spaces, Kybernetica. 11 (1975) 326-334.

[14]. Ruchi Singh, Smriti Mehta and A D Singh, Fixed Point Result in Probabilistic Metric Space, Mathematical Theory and Modelling, Vol.3, No.6, 2013.

[15]. Binayak S. Choudhury, Pradyut Das, P. Saha, Some $\varphi$-contraction results using CLRg property in probabilistic and fuzzy metric spaces, Annals of Fuzzy Mathematics and Informatics

[16]. A. F. R. L. Hierro and W. Sintunavarat, Common fixed point theorems in fuzzy metric spaces using the CLRg property, Fuzzy Sets and Systems 282 (2016) 131-142.

[17]. B. S. Choudhury and K. P. Das, A new contraction principle in Menger Spaces, Acta Math. Sin. (Engl. Ser.) 24 (2008) $1379-1386$.

[18]. J. X. Fang, On $\varnothing$ - contractions in probabilistic and fuzzy metric spaces, Fuzzy Sets and Systems267 (2015) 86-99.

[19]. M. Aamri and D. El Moutawakil, Some new common fixed point theorems under strict contractive contractions, J. Math. Analy. Appl. 270 (2002) 181-188.

[20]. L. Zadeh, Fuzzy sets Information and Control 8 (1965) 338-353.

[21]. W. Sintunavarat and P. Kumam, Common fixed point theorems for a pair of weakly compatible mappings in fuzzy metric spaces, J. Appl. Math. 2011, Art. ID 637958.

[22]. I. Kramosil and J. Michalek, Fuzzy metric and statistical metric spaces, Kybernetica. 11 (1975) 326-334.

[23]. M. Jain, K. Tas, S. Kumar and N. Gupta, Coupled fixed point theorems for a pair of weakly compatible maps along with CLRg property in fuzzy metric spaces, J. Appl. Math. 2012, Article ID 961210. 